\title{
Correction to: Technical specifications for an all-optical switch for information storage and processing systems
}

\author{
Ahmed Nabih Zaki Rashed ${ }^{1,2, a}$, Walid Fawzy Zaky ${ }^{2}$, Hazem M. El-Hageen ${ }^{1,3,4, b}$, \\ Aadel M. Alatwi ${ }^{3, c}$ \\ ${ }^{1}$ Renewable Energy \& Energy Efficiency Centre (REEEC), University of Tabuk, Tabuk, Saudi Arabia \\ 2 Electronics and Electrical Communications Engineering Department, Faculty of Electronic Engineering, \\ Menoufia University, Menouf 32951, Egypt \\ ${ }^{3}$ Electrical Engineering Department, Faculty of Engineering, University of Tabuk, Tabuk, Saudi Arabia \\ ${ }^{4}$ Egyptian Nuclear \& Radiological Regulatory Centre, Egyptian Atomic Energy Authority, Cairo, Egypt
}

(C) The Author(s), under exclusive licence to Società Italiana di Fisica and Springer-Verlag GmbH Germany, part of Springer Nature 2021

\section{Correction to: Eur. Phys. J. Plus (2021) 136:1100 https://doi.org/10.1140/epjp/s13360-021-01841-x}

In the original version of this paper, only one affiliation has been assigned to the author Ahmed Nabih Zaki Rashed, although two affiliations should have been assigned to him.

The correct affiliations are:

${ }^{1}$ Renewable Energy \& Energy Efficiency Centre (REEEC), University of Tabuk, Tabuk, Saudi Arabia.

${ }^{2}$ Electronics and Electrical Communications Engineering Department, Faculty of Electronic Engineering, Menoufia University, Menouf 32951, Egypt.

The original article has been corrected. We apologise for any inconvenience caused to our readers.

The original article can be found online at https://doi.org/10.1140/epjp/s13360-021-01841-x.

\footnotetext{
a e-mail: ahmed_733@yahoo.com (corresponding author)

b emails: hazemhageen@hotmail.com; helhageen@ut.edu.sa

c e-mail: aadel.alatwi@ut.edu.sa
} 